\title{
Correction to: Chemotherapy and Future Developments
}

lleana De Roma, Lucia Lombardi, and Gennaro Gadaleta-Caldarola

\section{Correction to: T. Scarabino, S. Pollice (eds.), Imaging Gliomas After Treatment,} https://doi.org/10.1007/978-3-030-31210-7_4

The original version of the chapter title "Chemotherapy Chemotherapy and Future Developments" was revised and has been corrected to "Chemotherapy and Future Developments".

The updated online version of this chapter can be found at https://doi.org/10.1007/978-3-030-31210-7_4 